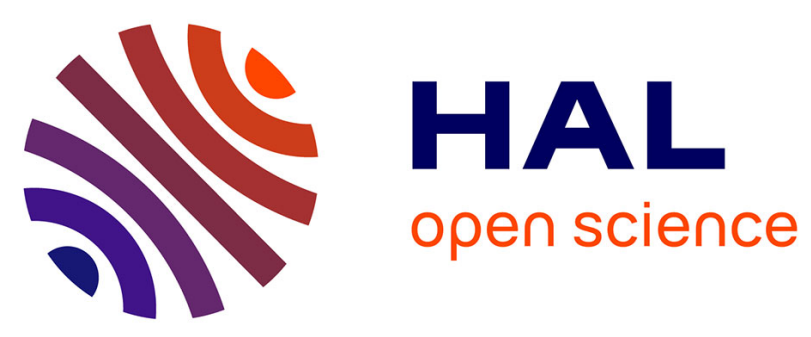

\title{
Persécution dedans/dehors
}

Fanny Dargent

\section{To cite this version:}

Fanny Dargent. Persécution dedans/dehors: De l'attaque du corps au projet de peau: étude d'un mouvement de projection détoxicant. Adolescence, 2008, Parano.., 3 (65), pp.681-695. 10.3917/ado.065.0681 . hal-01502996

\section{HAL Id: hal-01502996 https://hal.science/hal-01502996}

Submitted on 21 Jun 2017

HAL is a multi-disciplinary open access archive for the deposit and dissemination of scientific research documents, whether they are published or not. The documents may come from teaching and research institutions in France or abroad, or from public or private research centers.
L'archive ouverte pluridisciplinaire HAL, est destinée au dépôt et à la diffusion de documents scientifiques de niveau recherche, publiés ou non, émanant des établissements d'enseignement et de recherche français ou étrangers, des laboratoires publics ou privés. 


\title{
PERSÉCUTION DEDANS/DEHORS |
}

\author{
De l'attaque du corps au projet de peau : \\ étude d'un mouvement de projection détoxicant
}

FANNY DARGENT

Certains actes-symptômes rencontrés à l'adolescence sont à lire, audelà d'une classique organisation défensive, comme tentative de dégagement d'une situation de lutte interne qui se joue avant tout en termes de vie et de mort psychique plutôt qu'en termes de désir et d'interdit (même si ce qu'on appelle communément la frange névrotique peut être présente, prête à soutenir, dans le meilleur des cas, une « potentialité désirante » en germe dans la nouveauté pulsionnelle pubertaire). Bien que la destructivité domine largement ces tableaux cliniques " limites », la violence faite à soi-même peut cacher une recherche désespérée de maintenir vivants et soi et l'objet au sein d'un territoire interne ravagé, disputé par deux maîtres : la haine et le vide. Maintenir vivant relève avant tout des possibilités de conserver un minimum de respiration alors même que la circulation psychique souffre de dérégulation. L'histoire de Pauline ${ }^{1}$ illustre de façon dramatique l'aliénation morbide à l'autodestruction du corps propre, persécuté autant que persécutant, seule issue pour préserver le sentiment d'existence. Lutte a priori perdue d'avance contre ce « corps pour deux » (McDougall, 1989) qui tente de s'arracher à ce qui le fait vivre. Ici, la survie psychique en passe par la bruyance du corps et de ses organes dont le silence serait signe de mort, la sienne et/ou celle de l'autre, qu'elle vienne de l'intérieur ou de l'extérieur. La persécution du et par le corps est garant du sentiment de vivre. Aussi, on ne s'étonnera pas qu'aucune plainte ne vienne troubler ce déchaînement somatique, organes, chair et peau confondus.

1. Le temps de prise en charge thérapeutique avec Pauline a été largement étayé par leprécieux liens de travail avec les Docteurs Dominique Morel-Manela et Clémentine Rppaport ainsi que Cécile Bertin, éducatrice à l'Accueil Jeune, et l'équipe de l'unité dhospitalisation «Lits ados » au sein du service de pédopsychiatrie du Dr. Sylvain Berdah, Centre hospitalier Robert Ballanger d'Aulnay-sous -Bois (93).

Adolescence, 2008, 26, 3, 681-695. 
L'envahissement des troubles somatiques et autodestructeurs se fait enveloppe bruyante mais vivante, lit nécessaire et corporéisé des charges affectives de haine et de honte amassées depuis l'enfance. Au-delà de la présence envahissante de ce corps de souffrance ce sont les mots de Pauline qui ont pris le pas dès le début de nos rencontres puis ont continué à vivre dans un lieu où demeurent certaines rencontres cliniques attendant, peut-être, de renaître sur le papier, manière peut-être aussi de consolation face aux limites du traitement qu'opposent certaines organisations psychiques dont le paradoxe serait de maintenir une activation « déliaisante » continue pour maintenir la vie.

\section{UNE PARANOÏA DE CORPS ?}

Le mouvement que nous allons discuter se situerait entre «paranoïa ordinaire » à l'adolescence et « paranoïa somatique » (Tausk, 1975). Ilne s'agit bien évidemment pas de raisonner ici en termes de structure mais de dégager, dans un entre-deux où perdure, bien que de façon pathologique, la liaison corps/psyché, la projection massive à l'œuvre qui demeure en interne et en lisière, " à la limite » d'un hors soi peuplé d'objets aussi attracteurs que répulsifs.

Rappelons avec Ph. Gutton (2002), dans la lignée des travaux d'A. Green (1976) que le concept de limite n'a de sens qu'au regard des flux qui circulent de part et d'autre. «Il n'y a pas de dedans et de dehors mais des processus en cours $»^{2}$. Aussi, il va s'agir, dans l'après-coup de la prise en charge thérapeutique de Pauline, de ressaisir le processus de «tricotage psychique » de l'enveloppe dont les avatars ne s'appréhendent qu'au regard de la façon dont se sont effectués les aller-retour entre extérieur et intérieur et à l'intérieur même, entre les différentes instances. Les aiguilles pulsionnelles impulsent les mouvements figurant la qualité du maillage. La particularité de l'investissement du cadre thérapeutique en est le reflet tout autant que la spécificité de l'engagement transférentiel.

L'attaque du corps, qu'elle agisse en interne ou en surface, signe la dérégulation de la circulation psychique (sur-régime incorporatif/excorporatif au détriment de l'introjection et de la projection « hors soi ») allant de pair

2. Gutton, 2002, p. 94. 
avec un Moi-peau pathologique à double feuillet : l'un poreux, trop perméable aux excitations, insuffisamment différencié de l'objet, le second, carapace constituée dans un deuxième temps qui vise à « tout garder » menant à l'intoxication interne.

Le mouvement qui va nous intéresser concerne le passage d'une projection en interne (servant le déni du manque et de l'absence tout autant que l'évitement du commerce avec les objets externes trop effractants/attractants) et en surface à une projection « saine $»^{3}$, hors soi, d'allure phobique et persécutive, contemporaine à la constitution d'une frontière autonome ${ }^{4}$ (à condition de trouver des objets extérieurs 5 ayant fonction de réceptacle des contenus toxiques [Bion, 1962]). Les scarifications en sont la ligne médiane. Enrichie du point de vue topographique, l'effraction cutanée est à entendre, au-delà de la compulsion à répéter une effraction psychique/physique subie par l'objet (transformation d'un état de passivation [Green, 1999] en activation [Gutton, 2004])6, dans sa dimension d'expulsion hors de soi à visée détoxicante : la création de néo-orifices (Gutton, 2004) constitue une perforation à valeur de trous d'aération psychique face au psychisme

3. Que l'on peut rapprocher du « mouvement de projection protecteur » qui fait défut chez les femmes présentant des troubles des conduites alimentaires, décrit par C.Chabert dans le cadre de son hypothèse d'une version mélancolique du fantasme de séduction (Chabert et al., 2006. p. 68).

4. Nous rejoignons en ce sens l'approche de F. Ladame qui voit le processus d'agdolescence conme féproanisation structurale à travers le travail de désengagement la denstructia de barrières ou del imites (Ladame 1981, p. 7). Not e propos tend

cependant à mettre l'accent sur l'articulation entre approches topographique et psychodynamique. Chez Pauline, l'attaque du corps (entre autres du fait de la précocité de sa survenue pendant la période de l'enfance) ne peut de fait se suffire de la seule explication de l'attaque du corps en tant que rejet du corps sexué selon le modèle lauferien, c'est-à-dire pris dans la dimension fantasmatique de la relation aux objets préœdipiens et œdipiens. Le suivi de Pauline ouvre à un mode d'approche de l'attaque du corps qui tire vers l'autoconservatif en tant que toile de fond nécessaire au traitement du pubertaire. Une distorsion précoce des fonctions de contenance et de la circulation psychique en barre l'accès.

5. Ces objets extérieurs vont être d'autant plus susceptibles d'occuper cette fonction qils’s sont eux-mêmes contenus par un contenant-réceptacle: l'institution hospitalière a\&c ses d fférents services et interlocuteurs : corps vivant au sein duquel opère une criculation de représentations et d'affects « digérés » et élaborés quotidiennement grâce au travail de pensée et de liaison effectué en équipe. Le holding institutionnel opère comme un Moi-peau souple contenant pour contenir puis penser les attaques incorporatives et projectives des adolescents, assurant ainsi une fonction détoxicante.

6. Axe que nous avons travaillé dans « Les scarifications: du masochisme cruel aux scénarios pervers comme mouvement paradoxal de subjectivation » (Dargent, 2006). 
menacé d'asphyxie. Ces trous d'aération psychique se corporéisent en coupures sur la surface cutanée, contournant les orifices naturels ${ }^{7}$ permettant de soustraire temporairement l'adolescent au vécu d'intoxication interne et d'ouvrir à un mouvement de projection détoxicant.

\section{INCORPORATION MANIAQUE ET MÉLANCOLIE}

La psychothérapie de Pauline commence à la sortie d'une hospitalisation suite à une tentative de suicide ${ }^{8}$. Ce travail se poursuivra pendant près de deux ans, émaillé par deux autres hospitalisations, et nécessitera un cadre souple variant la fréquence des séances de une à cinq fois par semaine. Prise en charge extrêmement mobilisante du fait de la massivité transférentielle et des risques constants de passages à l'acte suicidaires et autodestructeurs. Le père, alcoolotabagique, est décédé deux ans auparavant des suites d'un cancer. La mère, ellemême suicidaire, est atteinte de troubles psychiques graves. Trois garçons aînés vivent loin de la famille. Pauline a été placée à l'âge de onze ans. À quatorze ans, elle fait une fausse couche après une première et brève relation amoureuse. Elle retourne vivre chez sa mère à l'âge de quinze ans, âge où elle décide d'effectuer une gastroplastie ${ }^{9}$ pour traiter l'obésité dont elle souffre depuis l'enfance. Des complications post-opératoires entraînent un coma et lui laisseront de nombreuses séquelles organiques. Elle retourne en convalescence chez sa mère avec qui elle nourrit une relation hautement pathologique marquée par un lien d'emprise et de grande dépendance, jusqu'à une tentative de suicide qui motivera une hospitalisation. Lors du début de la prise en charge, au sortir de l'hospitalisation, Pauline vit à l'hôtel après avoir accepté une prise en charge par l'Aide Sociale à l'Enfance (A.S.E.) afin de parvenir à une autonomie matérielle.

7. Les orifices naturels semblent ici demeurés fantasmatiquement possession

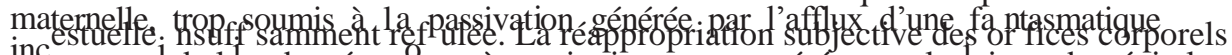
aú moment de là puberté, ouV ant à une jouissance tempérée sous le primat du génital, do.

it être sous-tendue par un minimum de souplesse au niveau de la respiration psychique alnt de pair avec la conscience d'une enveloppe corporelle comme surface contenant un volume (Anzieu, 1974).

8. La prise en charge des adolescents suicidants, étayée sur les travaux de F. Ladame, ma à l'instauration au sein de l'hôpital, depuis plus d'une décennie, d'un "protocole péventif » proposant une semaine d'hospitalisation en pédiatrie pendant laquelle se met en place un suivi éducatif et psychiatrique qui perdurera à la sortie de l'hospitalisation sous forme ambulatoire, au sein de l'Accueil Jeune. Pour Pauline, un suivi multifocal avait été ainsi mis en place: prises en charge psychiatrique, éducative, sociale et psychothérapique. C'est au cours de sa première hospitalisation que j'ai rencontré Pauline. C'est elle qui a demandé à ce que j'assure son suivi thérapeutique ambulatoire. La massivité transférentielle a eu pour effet la tendance au désinvestissement des liens avec les autres interlocuteurs, tendance contre-investie par le travail institutionnel.

9. Pose d'un anneau gastrique. 
Les pratiques d'incorporation ne sont pas nouvelles mais prennent, à cette période, une allure de précipitation maniaque que ce soit sur le versant de l'ingestion de toxiques (alcool, drogues, médicaments) ou celui de projets de corps (espoir d'une grossesse, projet d'une nouvelle gastroplastie). La thérapie est investie dans le même mouvement. Pauline vient à chacun de ses rendez-vous, parle beaucoup avec une extrême facilité, mêlant passé et actuel. Le clivage des parents en bon et mauvais (Bergeret, 1974) s'exprime d'emblée à travers une idéalisation sans faille pour le père disparu et une mère présentée ironiquement comme « une petite sainte ». À ce moment de rupture, la multiplication des incorporations d'objets comporte la visée illusoire d'installer des objets internes pour pallier la disparition de la perception des objets externes parentaux ${ }^{10}$, ne faisant que remplir un «puits sans fond ni forme ». L'extrême facilité de déplacement et de condensation des objets partiels externes incorporés vient dire l'urgence d'un recours au réel comme seule certitude d'avoir un « soi ». Au niveau économique, ces pratiques relèvent tant des procédés auto-calmants ${ }^{11}$ que de la compulsion à répéter indéfiniment l'effraction, source d'angoisse autant que palliatif à l'angoisse. Elles seraient aussi mise à l'épreuve dans le réel des capacités de contenance corporelle visant à établir une tridimensionnalité corporéisée « factice $»^{12}$. L'occupation de l'espace interne du corps par des objets réels serait autant de terrain gagné sur l'occupation par les objets fantasmatiques intoxicants. Tiercité illusoire, consistance donnée au caractère informe de l'angoisse, qu'elle soit de vide ou d'intrusion, objets concrets en place de la vacuité. Le clivage corps-mauvais/psyché-idéalisée (au service du déni de la perte et du manque) s'énonce de façon radicale : Pauline voit son avenir comme « déchéance de corps mais bien-être mental ».

INTOXICATION ET PERFORATION : «LES TROUS D’AÉRATION PSYCHIQUE »

Très vite, Pauline retourne vivre avec sa mère après avoir mis en échec les différents projets élaborés avec l'A.S.E. Les conflits reprennent. Les séances demeurent extrêmement riches sur le plan narratif. Les

10. $\mathrm{Ph}$. Gutton souligne l'absence «physique » de mois auxiliaires comme introduisant l'acte incorporatif (Gutton, 1984, p. 324).

11. Développés par les psychosomaticiens C. Smadja (1993) et G. Szwec (1993), ces prøédés visent à ramener le calme à travers la recherche répétitive de l'excitation. « Ceux qui les utilisent recherchent ce qu'on appelle la "détente" par des gomportements moteurs ou Bermetten de différencier ce qui apporte le calme de ce qui apporte la satisfaction (Szwec, 1998, p. 11).

12. En particulier en ce qui concerne l'anneau gastrique comme fétiche interne visant àafire l'économie de l'identification introjective au corps génital feminin dans sa dimens ón contenante ( « déni du vagin » mais surtout de « la complémentarité contenucontenant »en lien, selon Ph. Gutton aux pratiques d'incorporation [Gutton, 1984, p. 330]). Cet axe ne sera pas développé ici. 
silences de la mère sont sources d'angoisse pour Pauline qui reconnaît chercher le conflit afin de l'entendre à nouveau crier car c'est ainsi qu'elle la sent vivante. Chaque conflit entraîne une fuite dans sa chambre accompagnée de conduites d'autodestruction. Peu après, elle énonce un interdit maternel subi depuis l'enfance : interdit d'exprimer ses émotions, de pleurer. «Depuis, je garde tout. » Parallèlement se dégage l'image d'une enfant « objet » de la mère, fille unique ardemment désirée, gâtée à l'excès, et cependant inexistante à ses yeux. Face à ce double vécu d'interdit maternel et d'intenses sentiments de n'être rien pour elle, reste le corps pour exister. Corps obèse, attaqué depuis l'enfance (les scarifications ont commencé à l'âge de huit ans), sujet aux convulsions à l'âge de deux ans puis à de nombreux troubles respiratoires (bronchites chroniques, asthme...). L'autodestruction du corps se radicalise au moment de la puberté, dans une double dimension incorporative et excorporative à visée explusive : fausse couche, « sang vomi » lors des complications de la gastroplastie. Cette suractivation des mouvements incorporatifs/excorporatifs va de pair avec le défaut d'instauration d'un espace psychique autonome contenant au profit d'un envahissement par les objets internes ( «introjects hostiles hyperexcitants et dévorants »13) et externes. La nécessité de « tout garder », soumis fantasmatiquement à l'interdit maternel ${ }^{14}$, protège de la perte ${ }^{15}$ et des angoisses de vidage qui en sont la forme la plus archaïque, au risque de l'intoxication. Pauline dit être tel un sac, si elle pleure, elle se vide, « je ne pourrais plus tenir debout ». La représentation d'un Moi-sac est ici simple conteneur non pas de l'activité de penser mais d'une charge affective retenue qui organise le signifiant formel (Anzieu, 1987) : «Un sac vidé s'écroule » figurant les qualités psychiques de l'espace de Pauline : trop plein de contenus toxiques à valeur contenante préservant de l'effondrement narcissique, signifié ici par la perte de la tridimensionnalité. Tout garder plutôt que de risquer de tout perdre. Coincée entre incorporation narcissique de l'objet perdu et emprise interne basée sur une extrême soumission à la mère, d'un

13. Cahn, 1991, p. 109.

14. L'interditest ici référé au surmoi archaïque qui va de pair avec un écrasement topique.

15. Ce processus relève tant d'une problématique mélancolique (ici majorée par la perte réelle du père) que du travail du négatif, comme volonté de ne rien perdre et tentation d'immobilisme. 
registre plus auto-conservatif que libidinal, Pauline répond, face à l'urgence pulsionnelle, par le tout avaler/tout expulser. Le défaut d'introjection de la fonction contenante de l'objet externe (peau psychique [Bick, 1968]) entraîne une faillite de l'enveloppe pare-excitante puis la constitution d'une seconde peau carapace qui protège tant des angoisses de vidage que des projections agressives et destructrices. Le soulagement apporté par la pratique des scarifications s'explique par l'effet d'aération psychique produit par perforation de l'enveloppe carapace. «C'est ma façon de pleurer ${ }^{16}$ dira Pauline dans l'après-coup, lorsque cette défense morbide se révélera inefficace. L'activité toxique du Moi-peau ${ }^{17}$ opère ici, à défaut d'un possible recours au travail de liaison psychique, comme compromis de corps face à l'impasse psychique, frayant un chemin pulsionnel perverti hors soi mais qui demeure en lisière. La perforation de la surface cutanée agit comme appel d'air au cœur du vécu apnéique, entre figure de vide et figure de haine. L'insistance sur le ressenti de soulagement accompagnant les scarifications et la vue du sang qui coule ne peut se réduire à la dimension de décharge (n'importe quelle décharge motrice sans en passer par l'effraction pourrait alors faire l'affaire). Ce ressenti paraît sous-tendu par le caractère détoxicant de l'expulsion des charges affectives haineuses mais aussi par la relance d'un mouvement dans le réel venant, pour un bref temps, éloigner du vécu de mort psychique et d'immobilisme. À ce stade, les pulsions destructrices ne peuvent risquer une sortie hors soi vers l'objet au risque de le détruire, alors que la survie psychique en dépend, et de vider le moi de sa substance.

À l'approche des vacances d'été, après s'être elle-même enquise de mon absence, Pauline s'engouffre dans un nouveau mouvement d'incorporation maniaque réitérant les projets de corps. Les derniers

16. J. McDougall cite Henry Maudsely, psychiatre anglais : «The sorrow that has no vent in tears makes other organs weep. » («Quand le chagrin ne trouve pas d'issue dans les larmes ce sont d'autres organes qui pleurent. »), (McDougall, 1989, p. 254).

17. Dans la version de 1995, D. Anzieu supprime la neuvième fonction du Moi-peau, « fnction toxique» qualifiée d'anti-fonction en ce qu'elle relève du travail du « négatif ». Dans le gas de Pauline l'attaque du Moi-peau relève bien évidemment du travail de läeiner pemballement de la déliaison. Il s'agit d'une réorganisation à visée plus autoconservative que libidinale. Encore une fois : « solution la moins pire » face à la pression interne et externe. 
rendez-vous sont marqués par une recrudescence des conflits avec la mère et pour la première fois l'expression de vœux de mort à son égard dont Pauline dit chercher à se punir. La résurgence d'idées suicidaires suivie de deux journées d'errance avec prises de toxiques m'inquiètent suffisamment pour lui proposer une nouvelle hospitalisation qu'elle accepte.

\section{DE L'ESPACE CRU18 À L'ÉMERGENCE DE LA PERSÉCUTION}

Au retour de l'été, Pauline exprime des sentiments de vide. « Dans l'absence, on n'est rien »; « Je vous regarde penser ». L'objet externe est alors investi comme contenant et contenu dans une continuité de perception qui vient assurer un sentiment d'existence « prothèse » dont la contrepartie aliénante réside dans l'extrême dépendance et soumission où cela la place. Face à la confrontation à l'espace cru du fait de l'insuffisante inscription d'objets stables en l'absence de sa thérapeute, Pauline a effectué un intense mouvement de recollage à sa mère à forte tonalité incestueuse. «Je suis comme ma mère me veut : toujours là, immobile, inexistante. Au moins, elle est heureuse, non, pas heureuse, satisfaite. Quand ma mère est là, je ne pense plus. » Toute vie psychique semble s'effacer au profit de l'objet externe dans un mouvement d'identification adhésive entraînant une défaite pulsionnelle et l'abolition de l'espace transitionnel. Parallèlement à l'impact transférentiel, le travail de liaison se poursuit. La séparation de l'été a réactivé les souvenirs de séparations antérieures. Pauline rattache ses affects de tristesse à diverses représentations : le décès du père, ne pas être celle que sa mère aurait voulu qu'elle soit et l'intense culpabilité par rapport aux vœux de mort à l'égard de sa mère. Le mouvement mélancolique s'intensifie, elle « ne mérite pas d'être là, n'est rien ». Pour la première fois une position plus subjective se fait jour : «Je ne veux pas lâcher ma mère. » Pauline dit aussi, formulation de sa déception aux accents de vengeance, que parler ne sert à rien et qu'à présent elle préfère agir. Cet énoncé sonne comme une menace, amorce de ce qui va se jouer dans ce deuxième temps de la thérapie. Sa présentation physique a changé, elle vient parfois avec des lunettes noires, le visage dur et fermé. La réactualisation transférentielle

18. J. Doron dans Anzieu et al., 1987, p. 202. 
de la séparation va ouvrir à un puissant mouvement d'identification projective. Lors des silences de la mère, Pauline est certaine que celle-ci nourrit d'intenses vœux de vengeance à son égard. «Ça me fait peur mais c'est agréable à la fois de penser qu'elle pense à moi. » Une lutte intense s'engage pour ne pas exprimer les affects et représentations qui l'affleurent et l'ont animée pendant l'été. L'intensité de cette résistance, liée aux angoisses de vidage est aussi, non pas résistance à l'émergence des désirs inconscients mais farouche opposition à renoncer à une « volonté de soumission qui n'a d'autre raison qu'elle-même »19. En place de l'interdit maternel supposé s'érige le combat face à l'émergence de la colère et de la haine contre « les absents », la mienne de l'été, celle du père, et le sentiment d'avoir été trahie. Elle a lutté contre ses idées et s'est souvent scarifiée. L'attaque du corps se fait retournement des pulsions destructrices contre soi. S'ensuit un mouvement projectif, jusqu'alors pris en charge par le corps, dans un « hors soi » prenant deux directions : phobique et de persécution.

TRAVAIL DE LA HAINE20/TRAVAIL DU NÉGATIF : CONSTITUTION D'UNE FRONTIÈRE DEDANS/DEHORS

Pauline poursuit «sa lutte contre elle-même », résiste pour ne pas pleurer, passe des nuits à faire des jeux vidéo de combats « pour ne pas penser ». Les mouvements haineux se déploient à l'encontre de la mère au risque de l'hétéro-agressivité (elle a « vu rouge », a poussé sa mère, «a voulu la planter ») et d'un redoublement de l'autodestructivité (résurgence de somatisation : allergies et douleurs abdominales, tentative de scarifications sur le visage). Un mouvement agressif vite réfréné s'ébauche par rapport au père idéalisé ; pendant les séances, elle montre un agacement certain. Elle dit avoir toujours tout fait pour camoufler la haine au fond d'elle-même mais ne parvient plus à camoufler « ça ».

Dans le même temps, des affects de honte se lient aux représentations concernant l'intérieur de son corps dont « trop d'organes sont abîmés » tout comme sa voix, suite aux intubations. « Je ne veux pas

19. Green, 1993, p. 171.

20. Thème développé par C. Ternynck (2000). 
qu'on touche à mes cordes vocales ${ }^{21}$. Je ne veux pas que tout le monde voit dans mon estomac » énonce-t-elle avec des accents de rage et de détresse. L'apparition de la négation ${ }^{22}$ révèle la quête traumatophilique inconsciente (être effractée) tout en permettant de s'en dégager par l'instauration d'un mouvement de différenciation entre ce qui vient du dedans et ce qui vient du dehors, nécessaire à la constitution d'une frontière jusqu'alors incertaine. C'est par la négative qu'est nommé et donc attribué comme «à soi » un intérieur à partir duquel Pauline pose un double interdit de voir et de toucher ${ }^{23}$, permettant un décollement de la chose vers le mot. "La négation conjoint deux aspects opposés d'une destructivité jouissive et d'une symbolisation nécessaire à l'indépendance du moi. [...] On peut en déduire une théorie d'un rejet nécessaire de tout excès de chose pour qu'advienne un espace suffisamment évidé et dès lors voué à la pensée et à la parole ² $^{24}$

Au-delà du négatif, insistons sur la dimension persécutive sousjacente à la peur qu'on voie à l'intérieur d'elle. Il y a là un surinvestissement des pulsions scopique et de cruauté, pulsions, on l'a déjà souligné (Dargent, 2006), fortement mobilisées dans la pratique des scarifications. La sensation d'être transparente s'entend alors comme

21. Il y aurait beaucoup à dire sur l'investissement de l'organe vocal dont Pauline dira qu'est « le plus important pour elle ». Investi sur un mode narcissique dans la lignée dỉne identification au père, grand amateur d'opéra qui la soutena toute son enfance dans son désir de devenir chanteuse lyrique, puis indirectement " castrée » dans la lignée de la soumission aliénante à la mère. Perdre la voix, c'est régresser à l'infans.

22. Freud, 1925, pp. 135-139. Précisons que le contenu, ici, ne renvoie pas au refoulé imenscient (ce qui serait du registre de la dénégation névrotique) mais aux éléments cliés, insuffisamment symbolisés. La quête traumatophilique d'effraction appartient au registre de fonctionnement originaire et non primaire, sous-tendu par un mécanisme de clvage. Il ne s'agit pas là d'un fantasme inconscient mais de la répétition « actée » d'un vécu sensori-moteur précoce qui n'a pu être mis en représentation.

23. En référence aux travaux de D. Anzieu (1984). Nous ne pouvons développer ici lesenjeux pour l'organisation psychosexuelle de cet énoncé à valeur d'interdit qui vient « corriger » un défaut primaire concernant l'instauration du double interdit du toucher et, par suite du double interdit œedipien. Ici, l'interdit sous-entendu porterait sur la pulsion de cruauté dans sa dimension effractive, tant physique que psychique, renvoyant aux agoisses paranoïdes. Interdit d'accès à l'intérieur du corps et des pensées, de soi/de lautre (versant destructeur de la pulsion) d'où découle l'interdit protecteur du toucher par rapport à la mère dans sa dimension de séduction sexuelle 1 (versant sexuel de a aulsion) adolescent dans cette double dimension " d'opération primaire et d'organisation secondaire (la dénégation) ». «L'authenticitésubjective, enpasserait, [...] par des retours $\mathrm{ve}_{\mathrm{rs}}$ la négativité primaire, moments de mise en crise de la stabilité des frontières entre le moi et le non-moi » (Richard, 2001, p. 85). 
terreur d'être « retournée comme un gant », objet de « transpercement » 25 , livrée à une mère cruelle et effractive. Au cours de cette période de la thérapie, Pauline évoquera le souvenir terrifié de sa convalescence, à quinze ans, livrée, incapable de langage et de mobilité, aux soins maternels. Elle pourra me dire, alors que je ne l'y avais pourtant pas invitée, quelles représentations l'avaient animée à ces moments-là. Paradigme d'une scène incestuelle monstrueuse d' « un infans pubère » aux contours indifférenciés d'une mère toute-puissante.

À cette « reprise en main par le verbe » de l'espace interne liée aux affects de honte et aux angoisses intrusives, fait écho, dans la réalité, un mouvement phobique concernant l' « extérieur-externe »(Freud, 1923)26. Pauline s'isole dans le noir, ne sort plus de chez elle, l'extérieur lui fait peur car elle a l'impression que « tout le monde la voit comme [sa] mère : inexistante ». Dans le même mouvement, des persécuteurs sont désignés (les chirurgiens qui « l'ont ratée »; sa mère ; sa thérapeute de façon plus indirecte) signifiant la reconnaissance d'un objet extérieur différencié. L'extériorisation des pulsions destructrices envers la mère s'intensifie en même temps que se poursuit l'appropriation d'une position plus subjective. Elle s'oppose à sa mère, lui tient tête, ne veut plus être « une petite fille de $100 \mathrm{~kg}$ qui se tait ». Cette étape de la thérapie est le moment de bascule où la persécution ne sera plus subie au niveau somatique, de l'intérieur mais projetée dans un hors soi nécessaire àl'instauration d'une frontière dedans/dehors. Le déploiement des mouvements destructeurs accompagnant ce travail de décollement (d'arrachement) entraîne d'intenses sentiments dépressifs qui culmineront jusqu'à une nouvelle tentative de suicide ${ }^{27}$.

\section{PROJET DE PEAU ET QUÊTE IDENTITAIRE}

Ce passage à l'acte semble avoir été l'ultime tentative de maintenir une éternelle fusion avec les objets perdus. Plutôt que la mort, il ouvrira

25. Développé par P.-C. Racamier, « le fantasme de transpercement répond aux vœux de violer l'intimité corporelle et psychique de l'objet. Il ne s'agit que d'envahir, de pénétrer, de faire effraction; il ne s'agit que de prise et d'emprise » (Racamier, 1995, p. 86).

26. Reprécisé par F. Richard (2006). Ce terme permet d'insister sur le double processus à l'œuvre : à l'intérieur et entre extérieur et intérieur.

27. Cet « ultime » passage à l'acte sera lié par Pauline à l'histoire transgénérationnelle. 
le chemin d'Éros à travers l'entrée en scène d'un « courant sensuel » jusqu'à présent étouffé sous les charges pulsionnelles incestuelles et destructrices. Pour la première fois Pauline aborde la question de son orientation amoureuse, ne sachant si elle est attirée par les hommes ou les femmes. D'autres interrogations suivent plongeant racine dans le sexuel infantile autour de la différence des sexes et de ses identifications, étayé par les récits de nombreux souvenirs organisant une fantasmatique plus secondarisée. Elle se rapproche d'un ami et vit une période de bagarres et de petite délinquance, fortement dramatisée. Elle se promène avec une arme blanche qu'elle met en évidence lorsqu'elle entre dans le bureau, son portable sonne au cours des séances, elle montre une grande agitation préoccupée. Au-delà de la dimension provocante et persécutive, j'y vois l'importance, pour elle, de signifier qu'une « autre scène » se joue hors l'hôpital, dans cet « extérieur-externe » qui vient en reflet de la constitution d'un « extérieur-interne », soit un espace de refoulement protecteur. De l'extérieur entre dans l'intérieur du bureau, venant figurer une reprise de la circulation sans plus avoir à en passer par lesacrifice du corps. Et lorsque je lui exprime mon inquiétude alors qu'elle arrive à une séance en boitant après une bagarre qui a mal tourné, Pauline s'exclame d'un ton qui « laisse du jeu »: « Ce n'est pas moi cette fois ! » Le Moipeau se solidifie. La pratique des scarifications a disparu, Pauline évoque les cicatrices laissées par ses opérations puis, peu après, émerge un projet de tatouage figurant ses objets d'amour infantiles. Ce sera finalement l'un de ses frères qui lui tatouera, quelques mois plus tard, le visage d'une artiste. L'attaque de peau dans une confusion Moi-objet laisse place à la figuration des objets absents, poursuivant le travail de perte vers la mise en représentation au cœur d'un théâtre interne dont elle serait enfin, pour une part, maîtresse des lieux, tout en s'y inscrivant dans une filiation.

Par la suite Pauline engage différentes démarches à valeur de réparation, prises dans un ordre social symbolisé. Elle vit une relation amoureuse durable avec une jeune fille de son âge. Le suivi s'espace mais elle continue à venir de loin en loin, manière peut-être aussi de « vérifer » que nous avons survécu. L'appropriation d'un espace interne subjectivé lui permet de reconnaître et nommer certains traits de caractère. Elle se décrit comme jalouse, possessive, dépendante. La projection en interne et 
l'identification projective laissent peu à peu place aux projets de vie même si la tentation de la destructivité n'est jamais loin, ce qu'elle reconnait elle-même.

DE LA PERFORATION À LA FIGURATION : CICATRISATION DU MOI-PEAU POUR ENVELOPPER DES PENSÉES DE VIE

Nous avons essayé de montrer, à travers le suivi de Pauline, en quoi les attaques du corps, qu'elles soient internes ou externes, se proposent en garde-fou contre une pontentialité projective massive (psychotique) risquant d'altérer définitivement le rapport à la réalité et le lien aux objets, malgré toute la charge haineuse qui leur est associée. Le corps passionné se fait support de la haine dans un écrasement topique qui entraine un surinvestissement sensori-moteur. L'axe incorporatif/expulsif prend une large part et bien que demeurant sous l'emprise d'un narcissisme de mort, il paraît nécessaire à la survie psychique. L'investissement morbide de la surface de corps amorce un travail d'expulsion des forces destructrices tout en rendant possible un processus cicatriciel du Moi-peau (à condition d'un réceptacle capable de « survivre » et d'assurer une fonction détoxicante). Les scarifications prennent valeur de trous d'aération psychique avant que n'aient pu se développer, au cours du suivi, des mouvements projectifs phobique et persécutif, d'allure pathologique bien qu'ayant à ce moment valeur organisante au niveau topographique : renforcement d'une enveloppe contenante, différenciée (arrachée devrions-nous dire) du corps maternel, ouvrant à un travail d'appropriation subjective (Roussillon, 2006) qui en passe par le négatif (Freud, 1925 ; Green, 1993 ; Richard, 2001).

L'organisation défensive extrêmement coûteuse contre l'émergence pulsionnelle majorée par l'intrusion pubertaire et la réactivation des angoisses de perte, inscrite ici hors du modèle de la névrose infantile, implique massivement le corps qu'elle prenne la voie la plus passiverégressive de la somatisation ou celle, active, de l'autodestruction, quitte à en passer par l'utilisation d'objets extérieurs sollicités dans leur potentialité d'agents effracteurs (en particulier les chirurgiens). Les allerretour serrés, en circuit court, entre « décharge vers les profondeurs $»^{28}$

28. Green, 2002, p. 111. 
(somatisation) et « décharge hors psyché » ${ }^{29}$ (acting out) ont pu laisser place à des mouvements pulsionnels de plus grande envergure allant de pair avec la constitution d'une frontière contenante et la cicatrisation du Moi-peau, pouvant accueillir une image du corps érogène, support de l'activité de penser, soit un espace interne plus vivant détoxiqué de sa trop vive « folie pulsionnelle » (Cahn, 1991).

\section{BIBLIOGRAPHIE}

ANZIEU A. (1984). Le double interdit du toucher. Nouvelle Revue de Psychanalyse, 29 :

173-187.

ANZIEU D. (1985). Le Moi-peau. Paris : Dunod, 1995.

ANZIEU D. (1987). Les signifiants formels et le Moi-peau. In : D. Anzieu et al., Les

enveloppes psychiques. Paris: Dunod, 2003, pp. 1-22.

BION W. R. (1962). Aux sources de l'expérience. Paris : PUF, 2007.

CAHN. R. (1991). Adolescence et folie. Paris : PUF, 2004.

CHABERT C. et al. (2006). Actes et dépendances. Paris : Dunod.

DARGENT F. (2006). Les scarifications : du masochisme cruel aux scénarios pervers comme mouvement paradoxal de subjectivation. Adolescence, 24 : 651-663.

FREUD S. (1923). Le Moi et le Ça. In : Essais de psychanalyse. Paris : Payot, 1981, pp. 219-275.

FREUD S. (1925). La négation. In : Résultats, Idées, Problèmes II. Paris : PUF, 1985, pp. 135-139.

GREEN A. (1971). La projection : de l'identification projective au projet. In : La folie privée. Paris : Gallimard, 1990, pp. 195-223.

GREEN A. (1982). La double limite. In : La folie privée. Paris : Gallimard, 1990. pp. 293-316. GREEN A. (1993). Le travail du négatif. Paris : Les Éditions de Minuit.

GREEN A. (2002). Idées directrices pour une psychanalyse contemporaine. Paris : PUF. GUTTON PH. (1984). Pratique de l'incorporation. Adolescence, 2 : 315-338.

GUTTON PH. (2002). Violence et adolescence. Paris : In Press.

LADAME F. (1981). Les tentatives de suicide des adolescents. Paris : Masson.

LAUFER M., LAUFER E. (1989). Adolescence et rupture du développement. Une perspective psychanalytique. Paris : PUF.

McDOUgall J. (1989). Théâtre du corps. Paris : Gallimard, p. 254.

RACAMIER P.-C. (1995). L'inceste et l'incestuel. Paris: Les Éditions du collège. RICHARD F. (2001). Le processus de subjectivation à l'adolescence. Paris : Dunod. RICHARD F. (2006). La subjectivation : enjeux théoriques et cliniques. In : F. Richard,

S. Wainrib, La subjectivation. Paris : Dunod, pp. 81-121. 
SMADJA C. (1993). À propos des procédés autocalmants du moi. Revue Française de Psychosomatique, 4 : 9-26.

SZWEC G. (1993). Les procédés autocalmants par la recherche de l'excitation. Les

galériens volontaires. Revue Française de Psychosomatique, 4 : 27-51.

SZWEC G. (1998). Les galériens volontaires. Paris : PUF.

TAUSK V. (1975). De la genèse de «l'appareil à influencer» au cours de la schizophrénie.

In : Euvres psychanalytiques. Paris : Payot, pp. 177-217.

TERNYNCK C. (2000). L'épreuve du féminin à l'adolescence. Paris : Dunod.

Fanny Dargent

Équipe de Recherches sur l'Adolescence

Université Paris VII - Denis Diderot

UFR Sciences Humaines Cliniques

26, rue de Paradis

75010 Paris, France

Centre Hospitalier Intercommunal Robert Ballanger

Service de Pédopsychiatrie

93602 Aulnay-sous-Bois Cedex, France

fanny.dargent@aliceadsl.fr 